\title{
Robotic Hand Controlling Based on Flexible Sensor
}

\author{
Süleyman Bilgin ${ }^{a}$, Yavuz $\ddot{U}_{\text {ser }}^{b^{*}}$ and Muhammet Mercan ${ }^{c}$ \\ ${ }^{a, b, c}$ Akdeniz University, Faculty of Engineering, Electrical and Electronics Engineering, Antalya, Turkey \\ ${ }^{*}$ E-mail address: yuser@akdeniz.edu.tr \\ Received date: November 2016 \\ Accepted date: December 2016
}

\begin{abstract}
Today's technology has increased the interest in robotic systems and increase the number of studies realized in this area. There are many studies on robotic systems in several fields to facilitate human life in the literature. In this study, a robot hand is designed to repeat finger movements depending upon flexible sensors mounted on any wearable glove. In the literature, various sensors that detect the finger movement are used. The sensor that detects the angle of the fingers has been shown to provide high accuracy although cheap in present research. Moreover, by entering the sensor data into the open source interface program called Blender 3D, it can be seen on the program how the hand of the robot moves. Thanks to the prototype of robotic hand in this study is developed with different materials and mechanisms, it is possible to carry out experimental studies at low cost in places where it is unsuitable for human health and safety. The angle data on the sensors and servo motor position information are transmitted through the RF $433 \mathrm{MHz}$ wireless module. The determinations related to robot hand control are performed with Arduino card.
\end{abstract}

Keywords: Flexible Sensor, Robotic Hand, Blender 3D

\section{Introduction}

The researches on robotics have increased thanks to emerging new technologies and work on this field has gained speed. Especially, the innovative researches based on robotics hand and arm designs have been realized in several research centers on the world since the 80 's. The first prosthetic robot hand that consisted of 3 fingers and 1 thumb is developed by Utah University and MIT in 1986. It was on the study of the mobility of construction-purpose machines [1]. The development of robotics focused on the number of fingers and object attitude in 90's. However, there has been no testing of any mobility in these years $[2,3]$. At the end of the 90 's, robotics began to show some differences with the materials made [4] and the NASA Johnson Space Center made a new robotic handout consisting of two sub-sets [5]. The first set was the capability of mobility used for manipulation and the second one was a set of steadily grip during the movement of a particular object [5]. A new robotics control system was introduced by Byoung $\mathrm{K}$ et al. in 2000. This system consisted of two steps: RIFDS (Resolved Inter-Finger Decoupling Solver) and RIJDS (Resolved Inter-Joint Decoupling Solver). Through the RIFDS process, they have worked on providing elements with nondiagonal stiffness that can be hardened relative to only the geometric object to be gripped and similarly providing flexible finger control and independently providing flexible angle servo control through RIJDS [6]. The studies done up to this time has focused on noise reduction, lightness, human finesse, simplicity, cheap, low power and easily controllable [7,8]. Bundhoo et al. have designed a prosthetic hand that would provide small movements thanks to the 4-degree flexibility with biomimetic prosthetic fingers using a SMA (Shape Memory Alloy) to create a smart muscle system 
[9]. Shadow Robot Company has designed a robotic hand that resembles the human hand with its 24degree free mobility in 2006 [10]. Zajdlik et al. made it possible to recognize the automatic attitude of objects using wavelet theory and artificial neural networks on EMG signals in their study [11]. Nowadays, more and more sensitive prostheses hands have begun to be designed by using microcomputers in robotics applications. A 4-fingered system was developed using sensors wireless feedback and 8-bit microprocessor by Ramiah et al [12]. Zhang T. et al. have designed an FPGA / DSP based prosthesis [13]. In later work, capacitive sensors have begun to be widely used in robotic hand applications. Thanks to such sensors, high precision hand prostheses have been developed in robotic work [14]. Kappassov et al. designed robotic hand with high sensitivity based on capacitive, piezoelectric, quantum effect, optical and barometric sensors [15]. The application area of systems that can animate hand movements is almost limitless. The hand is one of the most complex organs of human body. Therefore, many researches in this field are being done both in the field of medicine and engineering [16-21]. A similar robotic arm has been realized without wireless connection in the previous studies. But this reduces the flexibility of remote control [22]. The main purpose of this work is to develop the hardware and software of the system, which enables remote manipulation of five finger movements, which can be controlled remotely by human wearing gloves. The basic structure of the system is shown in Fig. 1. The main distinction of the system is that the angleresistance relationship is obtained with the flexible sensor for the detection of the human fingertip angle and that it can be seen what the robot hand is monitoring simultaneously on the 3D Blender interface. This device can be used easily in the fields of medicine, industry and defense industry owing to developments and new additions. In particular, it can be used in dangerous humanitarian works for human health and safety such as bomb disposal, private laboratory operations. Furthermore, thanks to that the system is attractive in terms of cost, it will enable the growth of commercial artificial robotic hand.

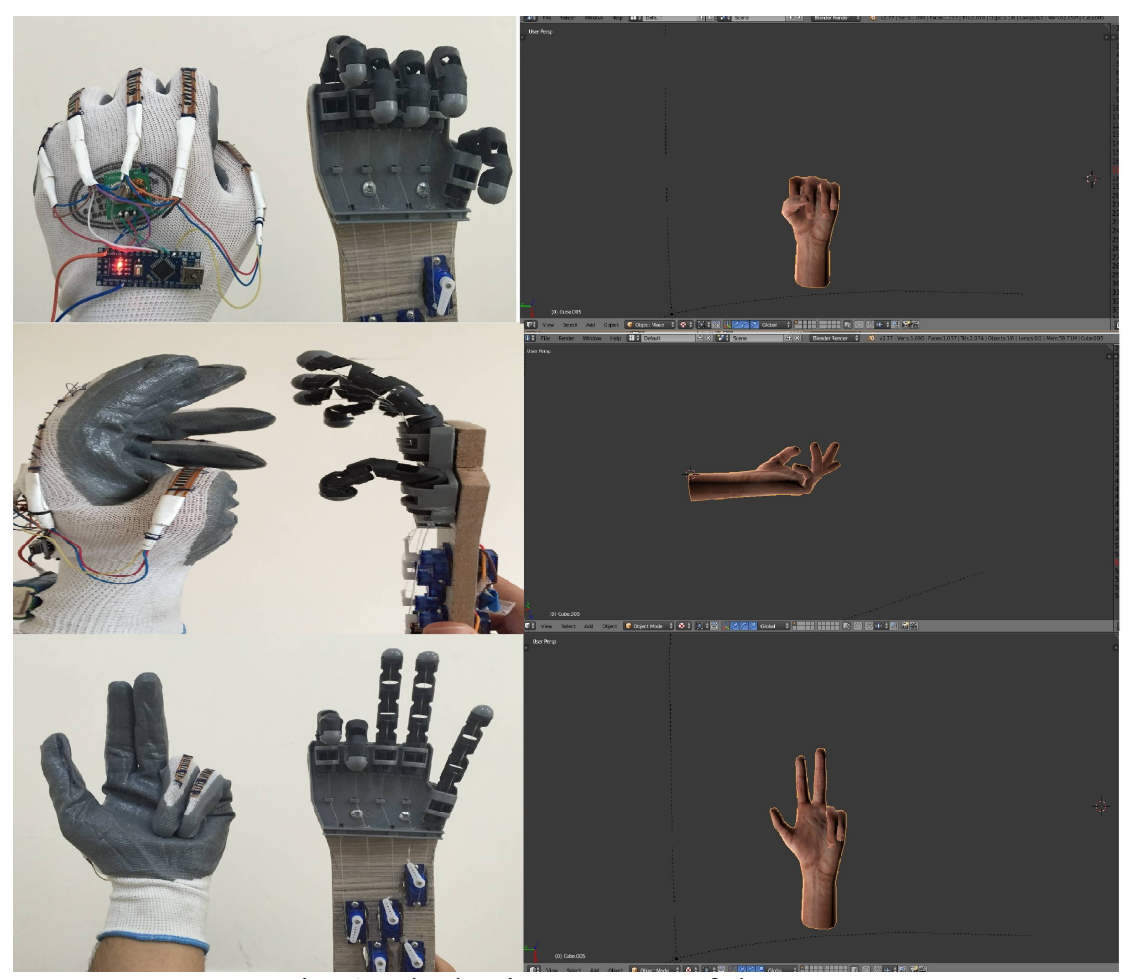

Fig. 1. The basic structure of the system

\section{Flexible Sensor}


The flexible sensors that can be produced as one-way or two ways flexibility are detectors whose resistance can be varied by the pressure applied on them. Since the human fingers can be twisted in one direction according to their nature, a one-way flexibility sensor is chosen as the sensor in this study. With the help of these sensors, servo motors can be controlled from $0^{\circ}$ to $180^{\circ}$. The relationship between the flexible sensors mounted on the glove and the servo motor angle values is shown in Table 1.

Table 1. Flexible Sensor Angle-Resistance Rates

\begin{tabular}{ccc}
\hline Finger & $\begin{array}{c}\text { Flexion } \\
\text { Angle }\end{array}$ & $\begin{array}{c}\text { Resistance } \\
\text { Value }\end{array}$ \\
\hline \multirow{2}{*}{$\mathbf{1}$} & $0^{\circ}$ & $\mathbf{1 3 . 4 k}$ \\
& $90^{\circ}$ & $\mathbf{1 6 . 5 k}$ \\
& $180^{\circ}$ & $\mathbf{2 0 . 1 k}$ \\
\hline \multirow{2}{*}{$\mathbf{2}$} & $0^{\circ}$ & $\mathbf{1 5 . 8 k}$ \\
& $90^{\circ}$ & $\mathbf{1 7 . 7 k}$ \\
& $180^{\circ}$ & $\mathbf{2 6 . 3 k}$ \\
\hline \multirow{3}{*}{$\mathbf{3}$} & $0^{\circ}$ & $\mathbf{1 3 . 7 k}$ \\
& $90^{\circ}$ & $\mathbf{1 7 . 2 k}$ \\
& $180^{\circ}$ & $\mathbf{2 3 . 6 k}$ \\
\hline \multirow{4}{*}{$\mathbf{4}$} & $0^{\circ}$ & $\mathbf{1 4 . 1 k}$ \\
& $90^{\circ}$ & $\mathbf{1 6 . 9 k}$ \\
& $180^{\circ}$ & $\mathbf{2 2 . 4 k}$ \\
\hline \multirow{5}{*}{$\mathbf{5}$} & $0^{\circ}$ & $\mathbf{1 4 . 3 k}$ \\
& $90^{\circ}$ & $\mathbf{1 8 . 6 k}$ \\
& $180^{\circ}$ & $\mathbf{2 4 . 1 k}$ \\
\hline
\end{tabular}

\section{The Connections of System}

The flexible sensors are mounted onto the glove as shown in Figure 2 to cover the entire length of the finger.

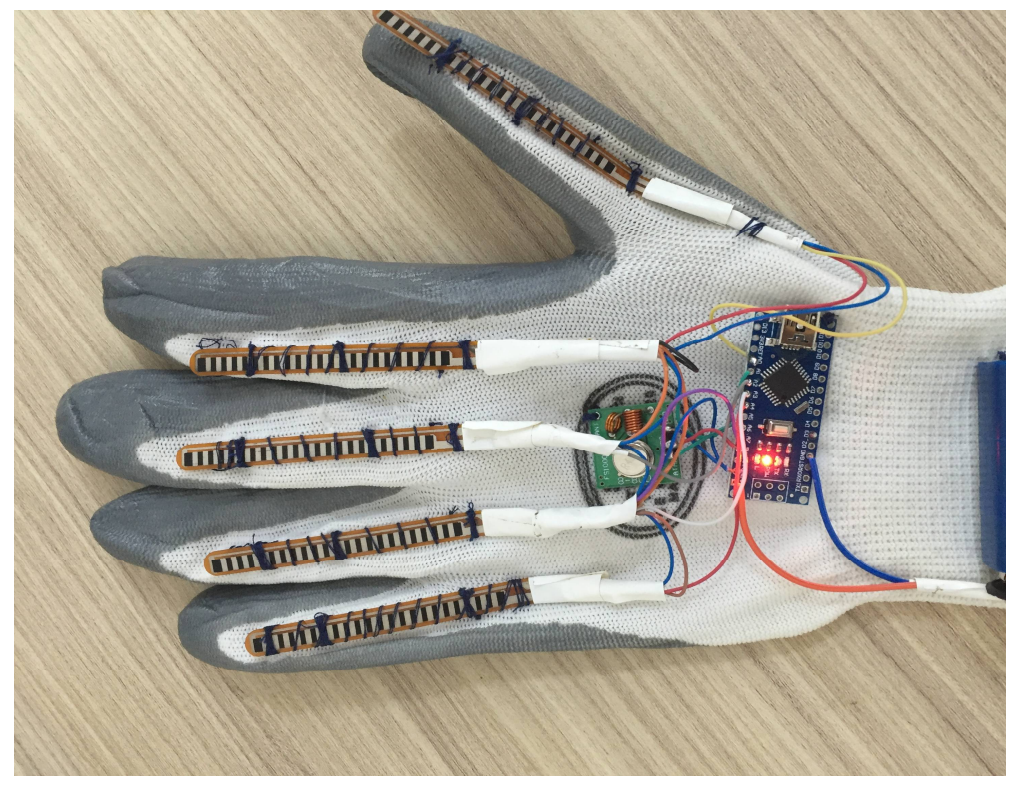

Fig. 2. The flexible sensor connections 
Figure 3 shows the flexible sensors and Arduino platform connections. Sensors are connected to the analog inputs of the microprocessor according to the voltage division principle.

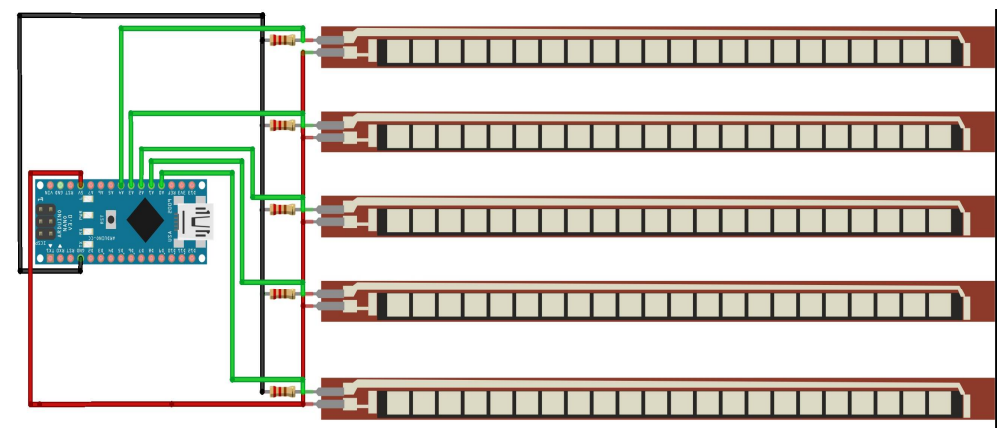

Fig. 3. Arduino flexible sensor connections

Micro servo motors whose advantages are the small size and high torques are used for robot hand movements. The torque is $1.8 \mathrm{~kg} . \mathrm{cm}$ for each servo motor used in the operation since it is performed only for motion animations. These torque values are sufficient for this study and can be selected easily as bigger values for other Power-requiring applications. Arduino servo motor connection is shown in Figure 4.

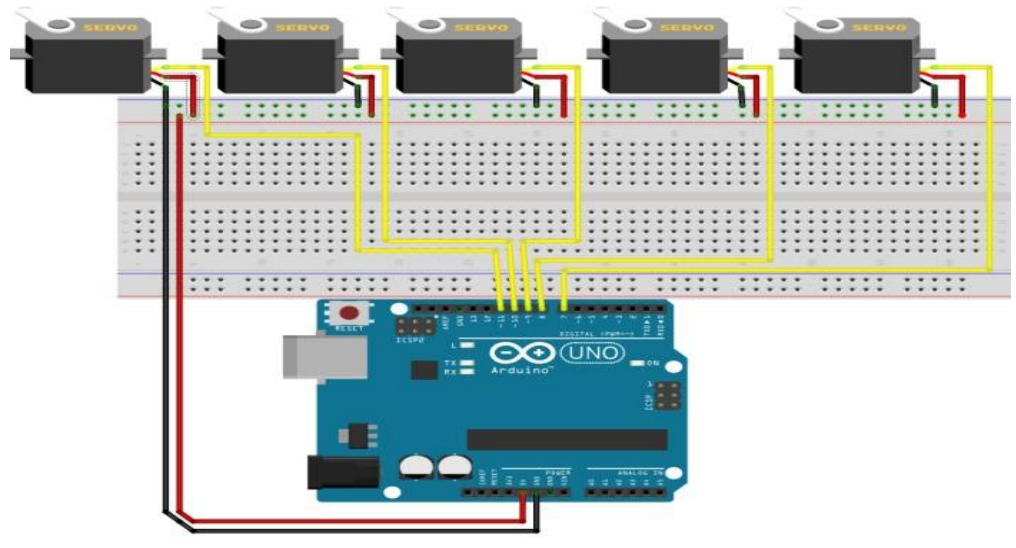

Fig. 4. Arduino servo motor connections

The analog information received from the finger sensors located in the fingers is converted to angle information in the digital form and then sent to remote robot hand through RF $433 \mathrm{MHz}$ wireless communication module. Figure 5 shows the RF $433 \mathrm{MHz}$ transceiver module and Arduino connections. 


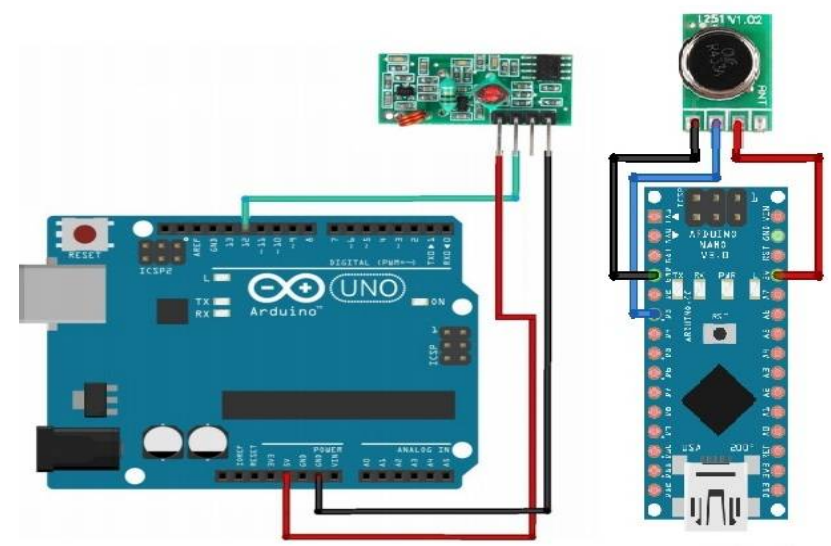

Fig. 5. Arduino RF $433 \mathrm{MHz}$ receiver connections

The information flow diagram for sending the values from the fingers up to the RF $433 \mathrm{MHz}$ transmitter module is shown in Figure 6.

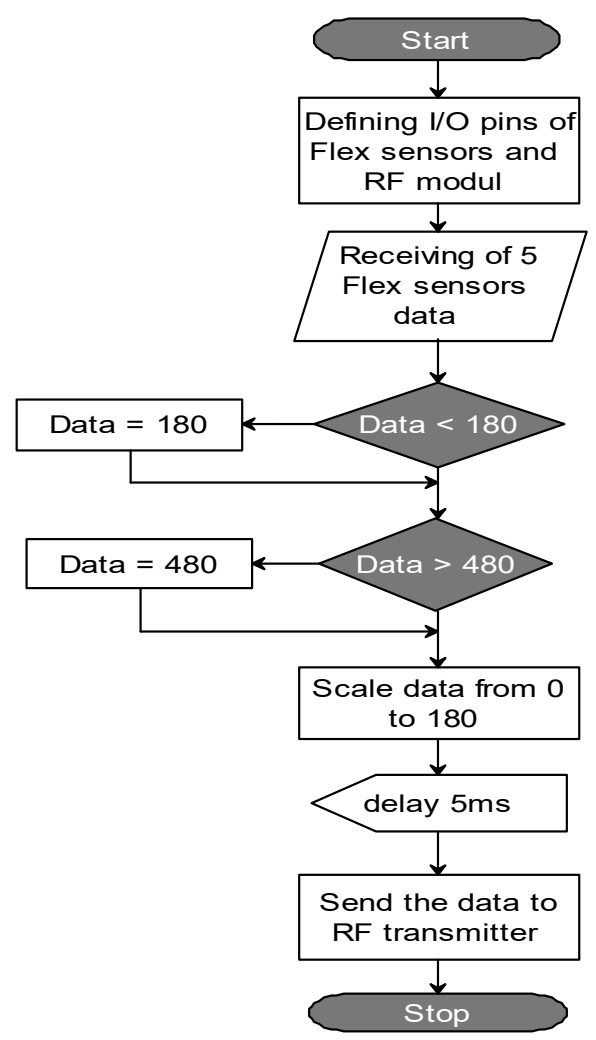

Fig. 6. Arduino data communication flow diagram of transmitter

The data from the RF $433 \mathrm{MHz}$ transmitter module is sent to the receiver module where it is decomposed and sent to the servo motors. The information flow diagram from the receiver module to the servo motors is shown in Figure 7. 


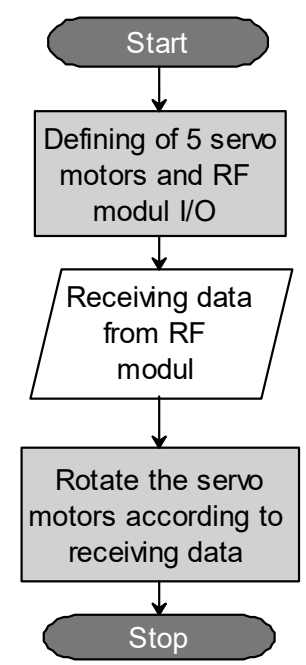

Fig. 7. Flow diagram of servo motor control

\section{Communication}

Wireless communication provides great convenience to receive information from mobile or stationary units that provide ease of movement or to send over a wide area. In this project, the RF $433 \mathrm{MHz}$ module, which is low cost and easy to find on the market, is used for wireless communication of glove and robot hand. The data from the five different flexible sensors are sent sequentially to the servo motors. A protocol for discrimination of this data on the receiver has been developed. The analog data received from the fingers are converted to digital values and then converted to angle values of the integer type. After converting angle values to char type, different angle values are assigned to the RF $433 \mathrm{MHz}$ transmitter module by combining different ASCII characters from the beginning of the angle values to distinguish the 5 different angle values. In this sequence, character "A" refers to "angle1", character "B" to "angle2", character "C" to "angle3", character "D" to "angle4" and character " $\mathrm{E}$ " to "angle5". This character string is then sent to the receiver module via the RF $433 \mathrm{MHz}$ transmitter module. The angle values that are received from the elements of this array are converted to integer values. The flow diagram of the receiver module is shown in Figure 8. 


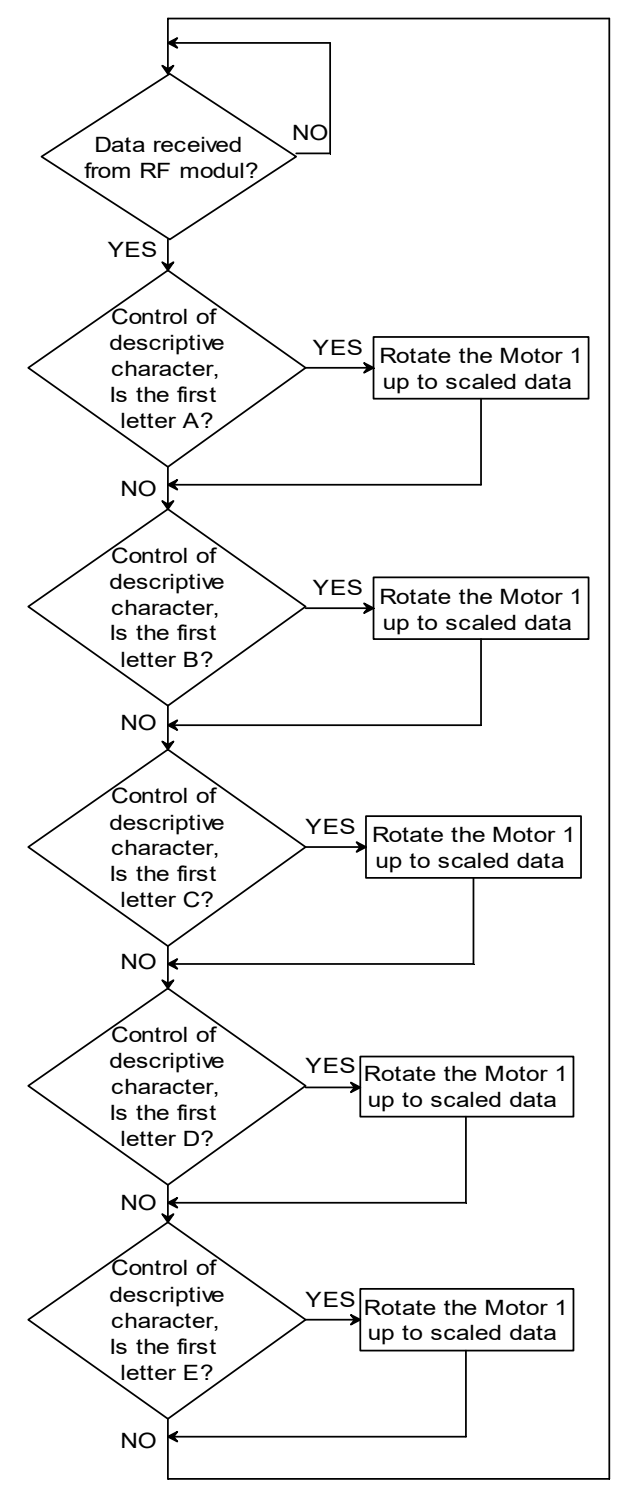

Fig. 8. Data communication flow diagram of receiver

\section{The Operation of System}

The analog angle values obtained from the flexible sensor are connected to the analog inputs of the Arduino Nano mounted on the glove. These angular values, which are converted to digital operation, are then sent to the Arduino UNO R3 platform, which is connected to the robot arm via the RF 433 $\mathrm{MHz}$ communication module. After these angle values are discriminated, they are sent to the servo motors by applying the pulse width modulation (PWM) technique. This operation is applied to 5 servo motors in the same way and the angle information is sent to the interface via the serial communication (USART). This operation is shown in Figure 9. 


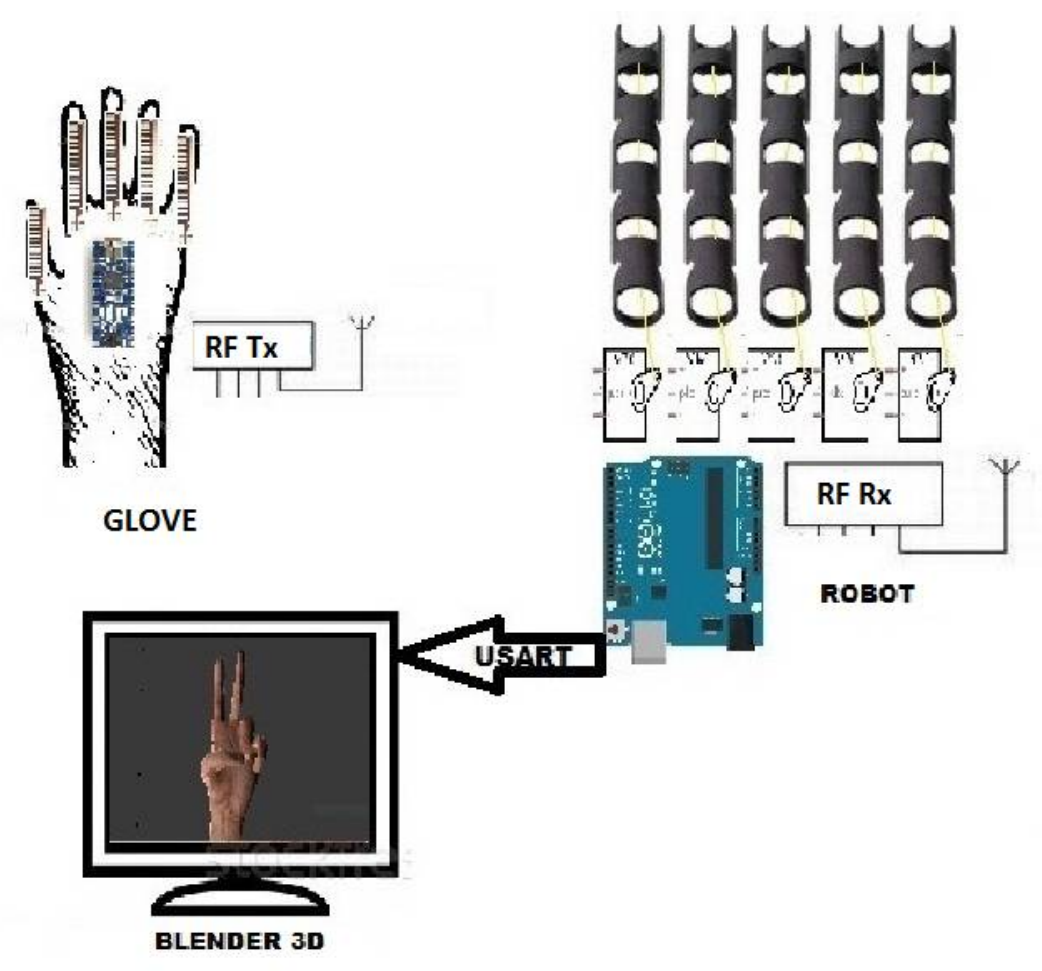

Fig. 9. Training of system

\section{Interface Design with Blender 3D}

Blender 3D is an open source free three-dimensional (3D) modeling and animation application. In addition to being a three-dimensional modeling animation software, it also includes a game engine, a video and sound montage software. In this project, in order to monitor the robot hand movements on the computer, the angle data is sent to Blender via serial communication and the finger movements are monitored instantaneously. In addition, real-time game control is provided through the interface that replicates finger movements, and animation work can be done on the computer. The interface of robot hand movements is shown in Figure 10. 


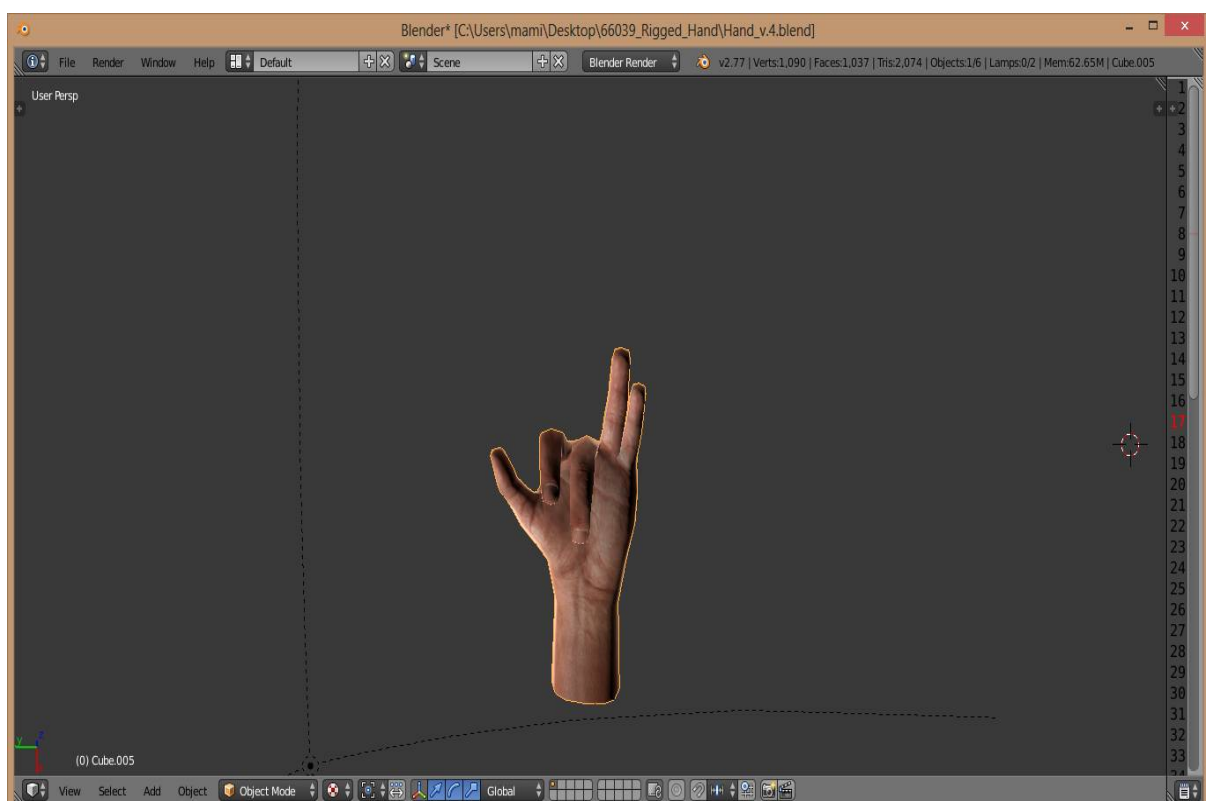

Fig. 10. Blender 3D interface

\section{Conclusions}

In this study, it has been observed that finger movements are perceived and imitated with high accuracy without any problems thanks to the flexible sensors mounted on any glove. The novelty of the system is that the system can be monitored on Blender 3D and wirelessly controlled via RF 433 module. With Blender 3D interface, the robot is able to track the position of the hand movements. Thanks to the RF $433 \mathrm{MHz}$ module, more comfortable movement is possible and the usage area is expanded. Since the servo motors high current at the start, the supplies are made separately. This system is possible that the robot hand will become commercially more widespread Since it is low cost. It can be also used in defensive industry, in bomb disposal works, in dangerous places in terms of human health and security, in animatronic works, in people who are living with discomfort at birth or later on their fingers. In the next study, wrist movements with flex sensors are also included in the study, and also EEG (Electroencephalograph) signals are used to control thought power.

\section{Acknowledgments}

The study has been supported by the Research Project Department of Akdeniz University, Antalya, Turkey. This study is a part of studies held by Akdeniz University Industrial and Medical Applications Microwave Research Center (IMAMWRC).

\section{References}

[1] Jacobsen, S.C., Wood, J.E., Knutti D. and Biggers K.B., Dextrous hand: Work in progress, Int J Robot Res., 321-350, 1984.

[2] Bekey, G.A., Liu H., Tomovic, R., et al. Knowledge-based control of grasping in robot hands using heuristics from human motor skills, IEEE Trans Robot Autom. 9,709-722, 1993.

[3] Landsberger, S., Shaperman, J., Setoguchi, Y., et al., Child prosthetic hand design: No small challenge, IEEE WESCON, 236-240, USA, 1996.

[4] Doshi, R., Yeh, C., LeBlanc, M., The design and development of a gloveless endoskeletal prosthetic hand, J Rehab Res Dev., 35, 388-395, 1998. 
[5] Ambrose, R.O., Aldridge, H., Askew, R.S., et al., Robonaut: NASA's Space Humanoid, IEEE Intel Syst. 15, 57-63, 2000.

[6] Byoung, K., Byung, Y., Hong, O., et al., Biomimetic compliance control of robot hand by considering structures of human finger, Proc IEEE Int Conf Robot Autom., 4, 3879-3886, 2000.

[7] Carrozza, M.C., Massa, B., Micera, S., et al., The development of a novel prosthetic handongoing research and preliminary results, IEEE/ASME Trans Mechatron., 7, 108-114, 2002.

[8] Massa, B., Roccella, S., Carrozza, M.C., Dario, P., design and development of an under actuated prosthetic hand, IEEE International Conference. Robotics and Automation, 4, 3374-3379, 2002.

[9] Bundhoo V, Park EJ. Design of an Artificial muscle actuated finger towards biomimetic prosthetic hand. Proceedings 12th International Conference. Advanced Robotics, ICAR'05 IEEE, 368-375, 2005.

[10] Zhao, D.W., Jiang, L., Huang, H., et al., Development of a multi-DOF anthropomorphic prosthetic hand, IEEE International Conference on Robotics \& Biomimetrics, 878-883, China, 2006.

[11] Zajdlik, J., The preliminary design and motion control of a five-fingered prosthetic hand, International Conference on Intelligent Engineering Systems. IEEE, London, United Kingdom, 2006. [12] Ramaiah, P.S., Venkateswara, R.M., Satyanarayana, G.V., A microcontroller based four fingered robotic hand, IJAIA, 2, 90-102, 2011.

[13] Zhang, T., Fan, S., Zhao, J., et al., Design and control of a multisensory five-finger prosthetic hand, Intelligent Control and Automation (WCICA), 11th World Congress, Shenyang, China, 33273332, 2014.

[14] Saikia, A., Mazumdar, S., Sahai, N., Paul, S., Bhatia, D., Verma, S., and Rohilla, P. K., Recent advancements in prosthetic hand technology, Journal of Medical Engineering \& Technology, 1-10, 2016.

[15] Kappassov, Z., Corrales, J.A., \& Perdereau, V., Tactile sensing in dexterous robot handsReview, Robotics and Autonomous Systems, 74, 195-220, 2015.

[16] Das, A.K., Das, R., Design and realization of prosthetic hand and its free movement, Int $J$ Emerg Technol Adv Eng., 3, 341-346, 2013.

[17] Pfeifer, R., Iida, F., Gomez. G., Designing intelligent robots on the implications of embodiment, J Robot Soc Jpn, 24, 9-16, 2006.

[18] Lee, S., Noh, S., Lee, Y., et al., Development of biomimetic robot hand using parallel mechanisms. IEEE International Conference on Robotics and Biomimetics, Guilin, Guangxi, China 2009.

[19] O’Toole, K.T., McGrath, M.M., Mechanical design and theoretical analysis of a four fingered prosthetic hand incorporating embedded SMA bundle actuators, Int $J$ Med Health Pharmaceut Biomed Eng., 1, 7, 2007.

[20] Olawale, J., Oludele, A., Ayodele, A., et al., Development of a microcontroller based robotic arm, Proc Compr Sci IT Educ Conf., 2007.

[21] Ciocarlie, M.T., Clanton, S.T., Spalding, M.C., Allen, P.K., Biomimetic grasp planning for cortical control of a robotic hand, Intelligent Robots and Systems IEEE/RSJ International Conference, 2271-2276, 2008.

[22] Omer Korkmaz, Mustafa Cem Kasapbasi, Robot Hand Making Copying Finger Motion, XVIII. Academic Informatic Conference, Turkey, AB 2016. 